A simplified, numerically verified model for the global plasma reaction on a local cooling

M. Z. Tokar, and M. Koltunov

Citation: Physics of Plasmas 20, 102502 (2013); doi: 10.1063/1.4823995

View online: https://doi.org/10.1063/1.4823995

View Table of Contents: http://aip.scitation.org/toc/php/20/10

Published by the American Institute of Physics 


\title{
A simplified, numerically verified model for the global plasma reaction on a local cooling
}

\author{
M. Z. Tokar and M. Koltunov \\ Institut für Energie- und Klimaforschung-Plasmaphysik, Forschungszentrum Jülich GmbH, \\ EURATOM Association, Trilateral Euregio Cluster, D-52425 Jülich, Germany
}

(Received 24 May 2013; accepted 3 September 2013; published online 2 October 2013)

An analytical model for heat losses along a magnetic surface to its small region cooled down abruptly to a very low temperature is deduced by applying a principle of minimum entropy production and verified by comparing with the results obtained by solving non-stationary two-dimensional heat conduction equation numerically. The model is elaborated further by taking into account heat flux limit and the plasma density modification due to the particle convection along the magnetic surface, triggered by the plasma pressure imbalance arising by the local cooling.

[http://dx.doi.org/10.1063/1.4823995]

\section{INTRODUCTION}

An abrupt cooling of small regions takes place under diverse conditions in plasmas of different types. This can happen, in particular, when impurity particles spread from intense localized sources in the edge plasma of fusion devices. As it was demonstrated in modeling, see Refs. 1 and 2, if the density of injected impurity neutrals exceeds a critical level of $10^{19}-10^{20} \mathrm{~m}^{-3}$, a thermal instability develops due to cooling of electrons and background hydrogen ions in inelastic excitation processes and in elastic coulomb collisions with impurity. As a result, the temperatures of plasma components drop abruptly, during a time in the micro-second range, from a value comparable with that before injection to a level of $1 \mathrm{eV}$.

As an example, we consider phenomena at the initial stage of massive gas injection (MGI) performed in tokamak devices to mitigate plasma disruptions. ${ }^{3-5}$ Observations show that the radiating impurity cloud spreads from the injection port in the toroidal direction with a velocity of $500 \mathrm{~ms}^{-1}$ being close to the sound speed of neutrals injected at the room temperature, see Fig. 15 in Ref. 5. On the one hand, this contradicts the assumption that radiating impurity ions produced by ionization of neutrals are heated to the temperature of the ambient plasma of $100 \mathrm{eV}$ and move away with a sound velocity of $20 \mathrm{kms}^{-1}$. On the other hand, the observed behavior can be explained by assuming a very low plasma temperature in the radiating impurity cloud cooled down as a consequence of a local thermal instability. Such an instability can indeed develop because by a typical MGI, the critical neutral density is achieved already during first $10 \mu \mathrm{s}$ after the moment when neutrals hit the plasma. Moreover, impurity ions generated in the neutral cloud and heated to a local temperature of $1 \mathrm{eV}$ cannot freely escape from the cloud with the corresponding sound speed because they are confined there by the friction force owing to elastic collisions with impurity neutrals and background ions. The latter are moving in the opposite direction to the cold impurity cloud, being accelerated by the pressure gradient arising with the plasma cooling in the cloud. ${ }^{1,2}$ Although direct measurements of local plasma parameters under MGI conditions are still absent, the formation of cold plasma in the neutral cloud has been experimentally demonstrated by a strong enough puffing of the working gas into tokamak TEXTOR. ${ }^{6}$ A very low plasma temperature in the radiating impurity cloud near the injection position allows also to interpret the rapid, during a time of $0.5-1 \mathrm{~ms}$ after the arrival of neutrals into the plasma, cooling of the whole plasma edge, see Fig. 2 in Ref. 3, Fig. 1 in Refs. 7 and 8. This edge cooling, occurring in spite of the radiation localization and low level of global radiation losses, can be caused by the electron heat conduction along magnetic field to the cold impurity cloud. ${ }^{9}$

In the present paper, we consider the evolution of plasma parameters in the region of magnetic surfaces beyond the cold plasma cloud on the stage of edge cooling. The extent of the cloud along the magnetic field increases during this event significantly less than by $1 \mathrm{~m}$ and is negligibly small compared with a characteristic field line length of $100 \mathrm{~m}$. Therefore, the heat conduction along the magnetic field towards the radiating impurity cloud plays the dominant role for the edge cooling. This phenomenon is of importance both for the later phases of thermal and current quenches during the plasma shutdown by MGI, being out of the scope of this study, as well as for other applications of impurity injection, e.g., for purposes of plasma diagnostics and confinement manipulation. ${ }^{10-13}$ Even such a limited task is challenging because the problem in question is non-stationary, multi-dimensional, and non-linear since heat conductions of plasma components are functions of the temperatures and their gradients. Recently, such a modeling has been done by solving numerically non-stationary two-dimensional heat conduction equations on a particular magnetic surface. ${ }^{9}$ Due to the non-linearity, essentially exaggerated through the very large temperature variation inside the calculation domain, the problem has to be treated with relatively small steps in time and space. This leads to very large CPU time expenditures, which will be again tremendously increased when, instead of, an isolated surface the whole plasma volume will be involved into consideration. Therefore, it is very desirable to find some appropriate description that would allow to reduce computation time significantly without any noticeable 
loss of the calculation accuracy. Furthermore, such a description has to be flexible enough to be extended by including phenomena, being too demanding for a direct numerical treatment, such as a heat flux limit (HFL) and the reaction of the plasma density on the local cooling. To develop an approach satisfying these requirements is the main task of the present study.

The rest of this paper is organized as follows. In Sec. II, we deduce from three-dimensional heat conduction equation, a one-dimensional one for the time evolution of the radial profile of the temperature averaged over the magnetic surface region beyond the radiating cloud of cold plasma near the injection port. An analytical model for the heat losses from this hot region to the cloud with the heat conduction along the magnetic field is elaborated in Sec. III. This model is verified in Sec. IV by comparing with the results of direct numerical solution for two-dimensional heat conduction equation obtained in the previous study in Ref. 9. The model is extended in Sec. V to include HFL and the reaction of the plasma particle density out of the cloud during the edge cooling phase. Finally, the results are discussed and conclusions are drawn in Sec. VI.

\section{BASIC EQUATIONS}

The time evolution of the temperature $T$ of a plasma component can be described by a non-stationary three dimensional heat transport equation

$$
1.5 \partial_{t}(n T)+\nabla_{r} q_{r}+\partial_{s} q_{s}+\partial_{l} q_{l}=Q-\Lambda
$$

where $n$ is the particle density of the plasma component in question; $q_{r}, q_{s}$, and $q_{l}$ are the components of the heat flux density in the radial direction $r$ across the magnetic surfaces, and on the magnetic surface perpendicular, $s$, and parallel, $l$, to field lines, respectively; $Q$ is the density of the volume heat sources and $\Lambda$-of the losses.

Consider the initial phase of a massive gas injection, where a significant edge cooling takes place. Since the injected impurity particles are still well localized near the injection position, the loss term $\Lambda$ can be neglected in the main region of magnetic surfaces beyond this localization area. The heat conduction coefficient, depending nonlinearly on the temperature and its gradient, varies extremely sharply by approaching to the cold impurity cloud, where the power is dissipated by impurity radiation. Therefore, very fine temporal and spatial resolution is required to get a converged solution of Eq. (1). Even with modern computers, a direct solving of this non-stationary three-dimensional equation with a reasonable accuracy is an extremely CPU time consuming task. One can, however, tremendously diminish computational efforts by reducing Eq. (1) to onedimensional equation describing the radial profile of the temperature averaged over the surface region in question, beyond the cold cloud. The cloud itself has been investigated in Refs. 1 and 2, where it was demonstrated that if the impurity neutral density exceeds a critical level, the plasma component temperatures drop very fast, during microseconds, from a value comparable with that before injection to
$1-2 \mathrm{eV}$. In the present paper, we consider the reaction of the plasma rest to such a prompt cooling near the injection position. By integrating Eq. (1) over the "hot" fraction of the magnetic surface, out of the cold cloud of radiating impurity, one gets

$$
1.5 \partial_{t}\left(A_{h}\langle n T\rangle_{h}\right)+A_{h} \nabla_{r}\left\langle q_{r}\right\rangle_{h}=A_{h}\langle Q\rangle_{h}-W_{c},
$$

where $A_{h}$ is the area of the "hot" magnetic surface region beyond the cross-section of the cold cloud, $\langle\cdots\rangle_{h}$ means the averaging over this area; during the edge cooling phase the cold cloud cross-section remains small compared with the whole magnetic surface and $A_{h} \approx 4 \pi^{2} r R$ with $R$ being the surface major radius. In Sec. III, an analytical model for the density per unit length in the radial direction of the heat loss along the magnetic surface to the cold cloud, $W_{c}$, is presented. In Sec. IV, this model is verified by comparing the results of numerical solution for the original heat transport Eq. (1) from Ref. 9 and the surface averaged one (2).

\section{MODEL FOR HEAT LOSSES TO THE COOLED AREA}

As it was already stressed above, we consider henceforth the regions on magnetic surfaces beyond the radiating cloud near the injection port, where the plasma is cooled down by direct interaction of electrons and main ions with the impurity. As experimental observations ${ }^{5}$ and calculations ${ }^{1}$ show, this cloud widens with the sound velocity of the injected impurity neutrals. During the edge cooling phase with a duration of $0.5-1 \mathrm{~ms}$, the cloud extend along the magnetic field, $2 l_{c}$, see Figure 1, increases insignificantly and remains small compared with the characteristic length $L$ of field lines on magnetic surfaces. Therefore, one can neglect the change of $l_{c}$, as well as of the cloud half-width $\delta_{c}$ during the whole process in question. The cross-section of the cold cloud by a magnetic surface, assumed as rectangle with the sides $2 l_{c}$ and $2 \delta_{c}$ in the $l$ and $s$ directions, respectively, remains a small fraction of the whole magnetic surface. For the majority of non-resonant magnetic surfaces, the stripe along the magnetic field connected to the cloud, see Figure 1(a), makes many turns in the toroidal and poloidal directions $x$ and $y$, respectively, before it intersects the cold area again. Rather than this happens, the temperature of the plasma component in question approaches its maximum value on the surface, $T_{h}$. The same temperature level is sustained on the middle dashed-dotted lines between the neighboring footprints of the stripe. The whole magnetic surface can be unfolded as a band between two middle lines, see Figure 1(b). At the outer boundary of this band, the temperature is equal $T_{h}$ and at the border of the cold area, $T_{c} \ll T_{h}$.

The task of this section is to interrelate the heat outflow along the magnetic surface to the cold cloud with the temperatures $T_{c}$ and $T_{h}$. To accomplish this, we use a method from Ref. 14 to find approximate solutions of partial differential equations of a parabolic type, such as heat conduction equations. This approach is based on the fact that such equations do allow neither periodic sign-changing solutions, nor a similar behavior of individual terms in the equation. Consider 
a

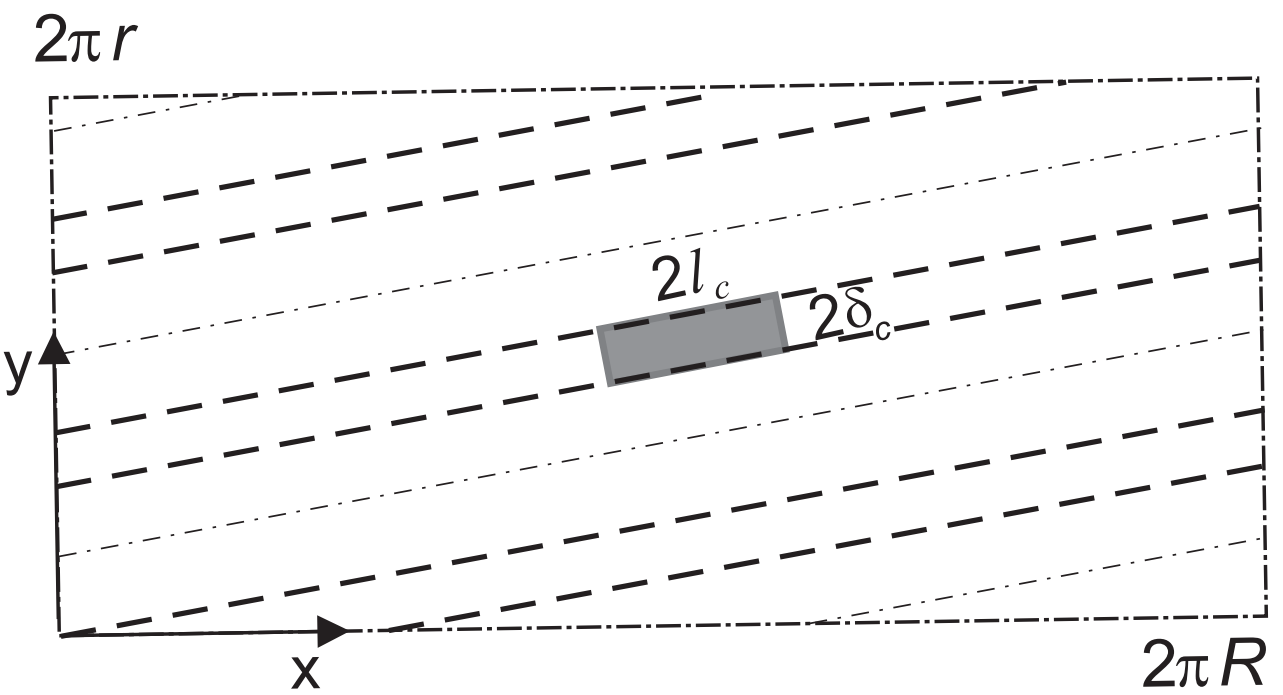

b $\Delta$

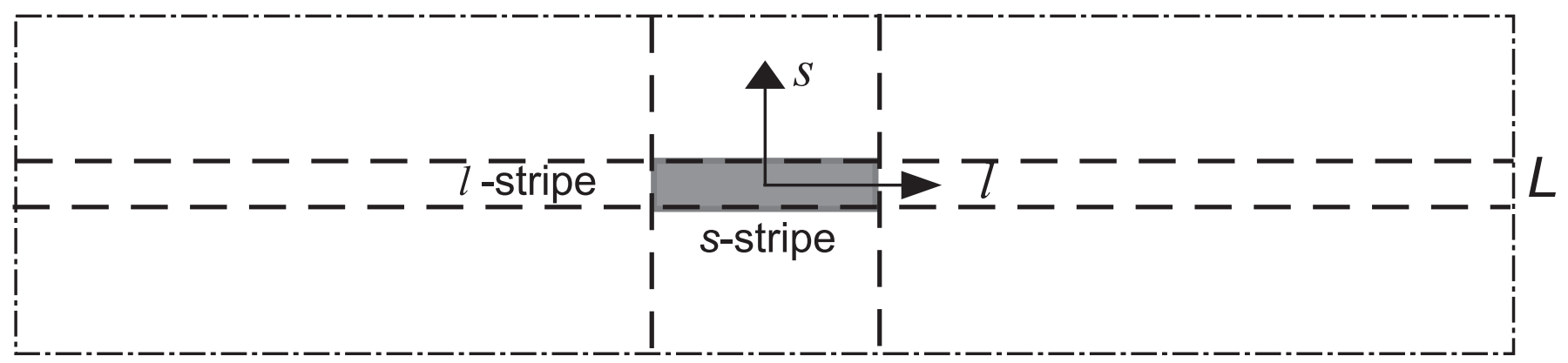

FIG. 1. A magnetic surface with the cross-section of the cold cloud, the grey area, and stripes connected to the area along the magnetic field (a); the surface unfolded as a band formed by lines running in-between two neighboring footprints of the parallel stripe.

the $l$-stripe in Figure 1(b) inside which the transport is dominated by the heat conduction along the magnetic field, $\kappa_{l}$, but convection losses can be neglected, see Ref. 9. The heat conduction Eq. (1) is written here in the form

$$
\partial_{l}\left(\kappa_{l} \partial_{l} T\right)=Q
$$

where the source term $Q_{l}$ on the right hand side combines all other terms, i.e., time derivative, transport in other directions, and so on. For the parallel heat conduction, we assume here the Spitzer-Härm approximation, ${ }^{15} \kappa_{l}=A T^{5 / 2}$, with $A$ dependent as $1 / \sqrt{m}$ on the particle mass $m$ of the plasma component in question. At the ends of the $l$-stripe, the temperature is fixed: $T=T_{c}$ and $T=T_{h}$ for $l=l_{c}$ and $l=L$, respectively. Moreover, $\partial_{l} T=0$ at $l=L$ since $T_{h}$ is the maximum temperature on the magnetic surface. By assuming $Q$ weakly varying with the coordinate $l$, one can straightforwardly find an approximate analytical solution of Eq. (3). With the boundary conditions above $Q$ can be excluded, and for the temperature profile along the $l$-stripe, we get

$$
T_{l}=\left\{T_{c}^{7 / 2}+\left(T_{h}^{7 / 2}-T_{c}^{7 / 2}\right)\left[1-\left(\frac{L-l}{L-l_{c}}\right)^{2}\right]\right\}^{2 / 7} .
$$

For the total heat loss with the parallel heat conduction to the cold area, one obtains

$$
W_{c}=\frac{4 A}{7}\left(T_{h}^{7 / 2}-T_{c}^{7 / 2}\right) \frac{4 \delta_{c}}{L-l_{c}} .
$$

The transport on magnetic surfaces perpendicular to field lines, the direction $s$, is normally much weaker than the parallel one and is neglected henceforth.

To calculate $W_{c}$ one has to know the band length $L$. We estimate this by employing a principle of minimum entropy production. ${ }^{16}$ By neglecting viscous and friction forces, the volume density of the entropy production rate is given as follows: ${ }^{17}$

$$
\theta \approx \kappa_{l}\left(\partial_{l} T\right)^{2} / T^{2}
$$

To evaluate the total entropy production rate $\Theta$ on the part of the magnetic surface in question, beyond the cold area crosssection, we take into account that the parallel temperature gradient is the most intensive in the $l$-stripe. This results in

$$
\Theta \approx \frac{16}{7} A \delta_{c} \frac{T_{h}^{7 / 2}-T_{c}^{7 / 2}}{\pi^{2} r R / \Delta-l_{c}}\left(\frac{1}{T_{c}}-\frac{1}{T_{h}}\right)
$$

where the band length $L$ was expressed through its width $\Delta$, by taking into account that the band covers the whole magnetic surface, i.e., $\Delta L=\pi^{2} r R$. Obviously, $\Theta$ is decreasing with diminishing $\Delta$ and approaches its minimum for $\Delta=\delta_{c}$, 
i.e., when the $l$-stripe covers tightly the whole magnetic surface. In this case, $l_{c} \ll L$ and we have

$$
W_{c} \approx \frac{16}{7} \frac{\delta_{c}^{2}}{\pi^{2} r R} A\left(T_{h}^{7 / 2}-T_{c}^{7 / 2}\right) .
$$

\section{MODEL VERIFICATION}

The outlined above analytical model for the heat losses to the cold area is verified here by comparing the results of numerical solutions for Eqs. (1) and (2) for the conditions of massive gas injection in JET H-mode. ${ }^{5,8}$ In Ref. 9, Eq. (1) has been solved on the stage of edge cooling in the region beyond the radiating impurity cloud, where the heat loss term $\Lambda$ is still small. The combination of the radial transport term on the left hand side and of the heat source term on the right hand side, $P=Q-\nabla_{r} q_{r}$, was approximated analytically to diminish CPU time. This approximation was motivated by the fact that in the initial steady state, the radial transport and heating are in balance, i.e., $P=0$. As the impurity injection is initiated and the cold cloud with radiating impurity arises near the injection valve, the radial temperature profile changes because the radial transport tries to cope with the parallel heat conduction to the cloud. Eventually, on a magnetic surface at the edge such a modification can be considered as an additional heat source, increasing with the deviation of the temperature from its initial level $T_{0}$. In the first approximation, $P \approx \nu n\left(T_{0}-T\right)$ is assumed, with some frequency $\nu>0$. The study in Ref. 9 has demonstrated that the characteristic time for the temperature decay due to parallel heat losses depends very weakly on $\nu$ and, therefore, the exact form of $P$ is not of very importance. Finally, according to Ref. 9, for the conditions in question of hot enough edge plasma in JET, the coupling between electron and ion temperatures through coulomb collisions is very weak and can be neglected.

As one can see from Sec. III, in the toroidal geometry of a tokamak no exact boundary conditions can be posed to transport equations written in the coordinate system $(l, s)$. Therefore, we proceed to the toroidal and poloidal coordinates $x$ and $y$ with respect to which all physical quantities are periodic with periods $2 \pi R$ and $2 \pi r$, respectively. The transformation between two coordinate systems is straightforward, $l=x \cos \psi+y \sin \psi, s=-x \sin \psi+y \cos \psi$, where the pitch-angle $\psi$ between the magnetic field and the toroidal direction is assumed, henceforth, independent of $x$ and $y$. In such a case, if the convective heat flow is neglected, Eq. (1) results in the following one:

$$
\begin{aligned}
& 1.5 \partial_{t}(n T)-\cos ^{2} \psi \partial_{x}\left(\kappa_{l} \partial_{x} T\right)-\sin ^{2} \psi \partial_{y}\left(\kappa_{l} \partial_{y} T\right) \\
& -\sin \psi \cos \psi\left[\partial_{y}\left(\kappa_{l} \partial_{x} T\right)+\partial_{x}\left(\kappa_{l} \partial_{y} T\right)\right]=\nu n\left(T_{0}-T\right) .
\end{aligned}
$$

Although the behavior of plasma density was not explicitly considered in Ref. 9, two limit cases were analyzed there: (i) the particle motion is so slow that $n$ remains unaffected and (ii) the particle fluxes are intensive enough to maintain the plasma pressure very close to a constant value on the magnetic surface. Computations have shown that the electron temperature evolution is actually insensitive to the assumptions on the density behavior and only the former situation, with the constant density $n \equiv\langle n\rangle$, is treated in this section. With the approximations above, Eq. (2) is reduced to an ordinary differential equation for the time evolution of the maximum temperature on the magnetic surface, $T_{h}$

$$
1.5 d T_{h} / d t=\nu\left(T_{0} / \theta_{h}-T_{h}\right)-W_{c} /\left(A_{h} \theta_{h} n\right) .
$$

Here, $\theta_{h}$ interrelates $T_{h}$ and the temperature averaged over the hot area of the magnetic surface, $\langle T\rangle_{h}$

$$
\theta_{h}=\frac{\langle T\rangle_{h}}{T_{h}} \equiv \frac{\int_{l_{c}}^{L} T d l}{T_{h}\left(L-l_{c}\right)} .
$$

By using the temperature profile from Eq. (4) under the conditions $T_{c} / T_{h}, l_{c} / L \ll 1$ in question, one gets $\theta_{h} \approx 0.95$.

Calculations are done for the conditions of experiments with MGI performed on the tokamak JET. ${ }^{5,8}$ The time evolution of the temperature near the separatrix with $R=3 \mathrm{~m}$, $r=1 \mathrm{~m}$, and $\psi=0.056$ is modeled; the initial electron temperature and density are assumed as in JET shot 77808 with MGI into the H-mode plasma, see the red curves in Figure 2 of Ref. $5, n_{0}=10^{19} \mathrm{~m}^{-3}$ and $T_{0}=250 \mathrm{eV}$. The dimensions of the impurity neutral jet cross-section by the magnetic surface are of $0.1 \pi r$ in the poloidal and toroidal directions. ${ }^{5}$ The plasma component temperatures in the cloud, $T_{c}$, are assumed, according to Refs. 1 and 2, equal to $1.5 \mathrm{eV}$.

Figure 2 demonstrates the time evolution of the maximum electron temperature $T_{e h}$ on the magnetic surface in question calculated with the Spitzer-Härm approximation ${ }^{15}$ to the parallel heat conduction, $\kappa_{l}=A T^{5 / 2}$, for the nominal

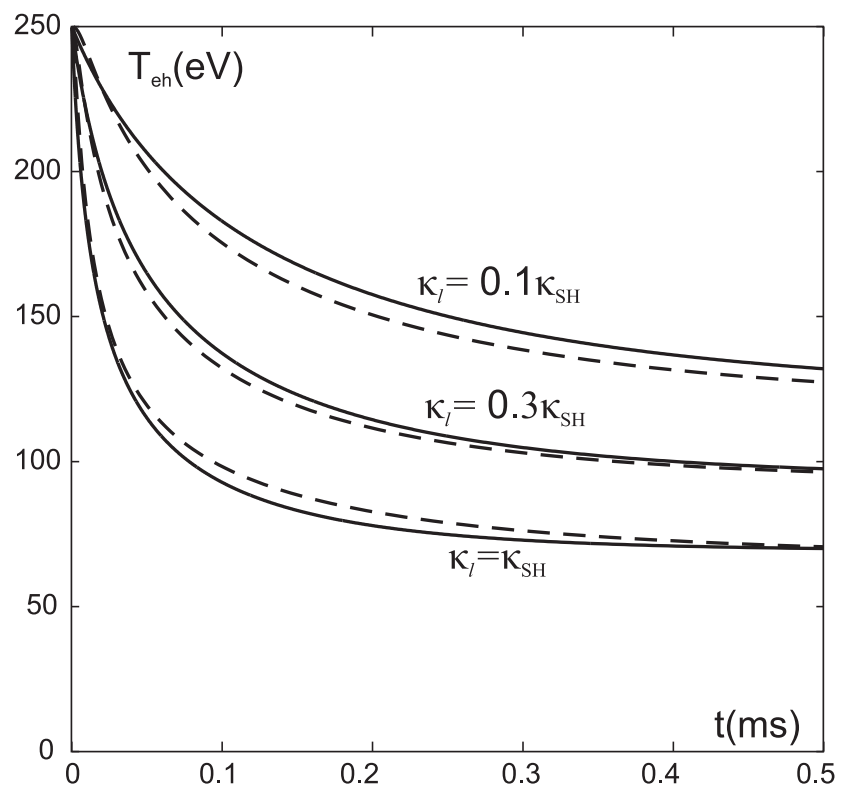

FIG. 2. The time evolution of the maximum electron temperature on the magnetic surface with the initial value $T_{0}=250 \mathrm{eV}$, computed for $\nu$ $=1000 \mathrm{~s}^{-1}$ with different assumptions on the parallel heat conduction, by using the elaborated analytical model for heat losses to the cold area (solid curves) and by solving two-dimensional heat transport equation numerically, see Ref. 9 (dashed curves). 
for electrons $A \approx 10^{18} \mathrm{~m}^{-1} \mathrm{~s}^{-1} \mathrm{eV}^{-2.5}$, and $A$ reduced by the factors 0.3 and 0.1 . One can see that the two-dimensional partial differential Eq. (6) and ordinary differential Eq. (7) provide very close time evolution of $T_{e h}$. This fact is very promising because the integration of Eq. (7) with the analytical model for heat losses to the cold cloud requires by a factor of $10^{4}$ smaller CPU time than the solution of Eq. (6) with a reasonable accuracy. We expect that also by calculations of the total radial profile evolution, caused by a local cooling at the plasma edge, the one-dimensional Eq. (2) can be used instead of the three dimensional one (1) without a significant loss of accuracy but with a similar relative reduction of the CPU time.

\section{FURTHER DEVELOPMENT OF THE MODEL}

\section{A. Introduction of heat flux limit}

The results above show that with the Spitzer-Härm approximation to the parallel heat conduction, $\kappa_{l}^{S H}$, the characteristic decay time for the electron temperature is of 100 $\mu s$, i.e., much smaller than that of $0.5-1 \mathrm{~ms}$ found in the experiments. As it was demonstrated in Ref. 9, a possible reason for this discrepancy is the violation of the condition for the applicability of $\kappa_{l}^{S H}$, requiring the characteristic scale for the change of the temperature $T$ along field lines, $L_{T}=T /\left|\partial_{l} T\right|$, much larger than the mean free path length between coulomb collisions, $\lambda_{c} \approx 10^{16} T^{2} / n,\left[\mathrm{~m}, \mathrm{eV}, \mathrm{m}^{-3}\right]$. Such a violation can happen in the region near the cold cloud with the extremely sharp temperature gradient and small $L_{T}$. In such a case due to the so called HFL phenomenon, one expects for electrons a heat flux density, being a small fraction $\xi$ of the free-streaming one, $n T^{3 / 2} / \sqrt{m_{e}}$. The fact that $\xi \ll 1$ is explained by (i) non-local effects in collisionless plasma, reducing the perturbation in the distribution function caused by a temperature gradient, ${ }^{18}$ and (ii) braking of electrons by ambipolar electric field. ${ }^{19}$ By following an approximate formula in Ref. 20, describing smoothly the transition between collisional and collisionless limits, we obtain, instead of, the relation (5) the following one:

$$
W_{c}=\left[\frac{7 \pi^{2} r R}{16 \delta_{c}^{2} A\left(T_{h}^{7 / 2}-T_{c}^{7 / 2}\right)}+\frac{\sqrt{m_{e}}}{\delta_{c} \xi n_{c} T_{c}^{3 / 2}}\right]^{-1} .
$$

\section{B. Plasma density response to local cooling}

The drop of the temperatures of plasma components in the impurity cloud near the injection position caused by thermal instability due to radiation and coulomb collisions with impurity results in a large pressure gradient. This drives flows of the main plasma particles along magnetic field lines toward the cold cloud. As a result, the density of the main hydrogen ions in the cloud, $n_{c}$, increases and that in the "hot" region of magnetic surfaces, $n_{h}$, decreases. The plasma streams to the cold impurity cloud with a sound velocity of the main hydrogen ions, corresponding to the temperature in the "hot" surface area, which is much larger than the spreading velocity of the cold impurity cloud. Therefore, the cloud dimensions remain much smaller than the field line length $L$ during the whole process of the plasma density redistribution. To model properly this process one has to take into account particle and parallel momentum balance equations. At the plasma edge in the "hot" region of magnetic surfaces beyond the impurity cloud, they are taken in the following form:

$$
\begin{gathered}
\partial_{t} n+\nabla_{r} \Gamma_{r}+\partial_{s} \Gamma_{s}+\partial_{l} \Gamma_{l}=S, \\
\partial_{t} \Gamma_{l}+\nabla_{r}\left(\Gamma_{r} \Gamma_{l} / n\right)+\partial_{s}\left(\Gamma_{s} \Gamma_{l} / n\right) \\
+\partial_{l}\left[\Gamma_{l}^{2} / n+n\left(T_{e}+T_{i}\right) / m_{i}-\eta_{l} \partial_{l} \Gamma_{l}\right]=F,
\end{gathered}
$$

where $S$ is the plasma source density due to recycling of main neutrals since the contribution due to ionization of the injected impurity gas is small in the region in question; $T_{e}$ and $T_{i}$ are electron and ion temperatures, respectively, $m_{i}$ is the mass of the hydrogen isotope ions, $\eta_{l}$ their parallel viscosity and $F$ is the sum of other forces such as friction with recycling neutrals.

The integration of Eq. (10) over the hot region of the magnetic surface provides:

$$
\partial_{t}\left(A_{h}\langle n\rangle_{h}\right)+A_{h} \nabla_{r}\left\langle\Gamma_{r}\right\rangle_{h}=A_{h}\langle S\rangle_{h}-G_{c},
$$

where $G_{c}$ is the density, per unite length in the radial direction, of the loss of charged particles caused by the flow to the cold cloud. To find $G_{c}$, we neglect the contribution due to perpendicular transport on magnetic surfaces, which is normally much less intensive than that along the magnetic field. To estimate the latter, we write Eq. (10) as follows:

$$
\partial_{l} \Gamma_{l}=S_{l}
$$

where $S_{l}$ combines all other terms in Eq. (10). With the boundary conditions $\Gamma_{l}\left(l_{c}\right)=-\Gamma_{c}, \Gamma_{l}(L)=0$ and assuming a constant $S_{l}$, we get:

$$
\Gamma_{l}=-\Gamma_{c}(L-l) /\left(L-l_{c}\right), \quad G_{c}=4 \delta_{c} \Gamma_{c} .
$$

An equation for the density of the parallel outflow to the cold cloud, $\Gamma_{c}$, we obtain by integrating Eq. (11) over the hot region of the magnetic surface. For $l_{c} \ll L$ and the linear profile of $\Gamma_{l}(l)$ found above, one gets:

$$
\begin{aligned}
& \partial_{t}\left(A_{h} \Gamma_{c}\right)+A_{h} \nabla_{r}\left(\frac{\Gamma_{r} \Gamma_{c}}{n}\right) \\
& \quad \approx 2 \delta_{c}\left[\frac{n_{h}\left(T_{e h}+T_{i h}\right)-2 n_{c} T_{c}}{m_{i}}-\frac{\Gamma_{c}^{2}}{n_{c}}-\frac{\eta_{l} \Gamma_{c}}{L}\right] .
\end{aligned}
$$

Finally, we need an equation for the density of the main ions at the boundary of the cold impurity cloud, $n_{c}$. Roughly, this is governed by the balance of the main ions in the cloud

$$
\partial_{t}\left(\delta_{c} l_{c} n_{c}\right)+\delta_{c} l_{c} \nabla_{r} \Gamma_{r}=\delta_{c} l_{c} S+G_{c} .
$$

Note, due to the plasma quasi-neutrality $n_{c}$ can be significantly smaller than the electron density in the impurity cloud. ${ }^{1,2}$ This fact may be also of importance for $n_{c}$ itself because the electron density is involved into the source density $S$. 
To relate the densities $\langle n\rangle_{h}, n_{h}$, and $n_{c}$, we take into account that in any point beyond the impurity cloud, for $l_{c} \leq l \leq L$, the plasma density is between the minimum level $n_{h}$ far from the cloud and the maximum local value, which is approached in the equilibrium state with a constant pressure, $n_{m}(l)=\eta(l) n_{h}$, where $\eta=\left(T_{e h}+T_{i h}\right) /\left(T_{e l}+T_{i l}\right)$ and $T_{e l}, T_{i l}$ are given by Eq. (4) for electrons and main ions, respectively. In the first approximation, we assume that $n(l)$ is a linear combination of $n_{h}$ and $n_{m}(l)$, with the individual weights defined from the conditions $n\left(l=l_{c}\right)=n_{c}$ and $n(l=L)=n_{h}$ :

$$
n(l)=n_{h} \frac{\eta\left(l_{c}\right)-\eta(l)}{\eta\left(l_{c}\right)-1}+n_{c} \frac{\eta(l)-1}{\eta\left(l_{c}\right)-1} .
$$

With a definition of $\langle n\rangle_{h}$ similar to that for $\langle T\rangle_{h}$, see Eq. (8), one gets for $T_{c} \ll T_{e h, i h}$

$$
\langle n\rangle_{h} \approx n_{h}+0.16 n_{c} T_{c} /\left(T_{e h}+T_{i h}\right) .
$$

Thus, even if $n_{c}$ is approaching its maximum level $2 n_{h} T_{c} /\left(T_{e h}+T_{i h}\right)$, corresponding to the pressure balance, the deviation of $\langle n\rangle_{h}$ from $n_{h}$ is less than $10 \%$. Therefore, an error made by prescribing $n(l)$ in the form of Eq. (15) is, probably, insignificant. For the averaged pressures of plasma components, involved into Eq. (2), we have obtained

$$
\langle n T\rangle_{h} \approx\left[0.95 n_{h}+0.12 n_{c} T_{c} /\left(T_{e h}+T_{i h}\right)\right] T_{h} .
$$

\section{Results of calculations}

The functioning of the full system of Eqs. (2) and (12)-(14) is demonstrated in the same approximation for the combination of the radial transport and source terms as in Ref. 9, by assuming these proportional to the deviation of the parameters $T_{e h}, T_{i h}, n_{h}, \Gamma_{c}$, and $n_{c}$ from their initial values $T_{0}$, $T_{0}, n_{0}, 0$, and $n_{0}$, respectively, we obtain from Eqs. (12)-(14):

$$
1.5 d\langle n T\rangle_{h} / d t=\nu\left(\langle n\rangle_{h} T_{0}-\langle n T\rangle_{h}\right)-\left(W_{c}+10 \Gamma_{c} \delta_{c} T_{c}\right) / A_{h}
$$

for both plasma components,

$$
\begin{gathered}
d\langle n\rangle_{h} / d t=\nu\left(n_{0}-\langle n\rangle_{h}\right)-4 \delta_{c} \Gamma_{c} / A_{h}, \\
d \Gamma_{c} / d t=\frac{2}{L}\left[\frac{n_{h}\left(T_{e h}+T_{i h}\right)-2 n_{c} T_{c}}{m_{i}}-\frac{\Gamma_{c}^{2}}{n_{c}}-\frac{\eta_{l} \Gamma_{c}}{L}\right]-\nu \Gamma_{c},
\end{gathered}
$$

and

$$
d n_{c} / d t=\nu\left(n_{0}-n_{c}\right)+\Gamma_{c} / l_{c},
$$

where convection is also included into the heat losses to the cold cloud. To integrate Eqs. (16) and (17) straightforwardly, as equations for the variables $\langle n T\rangle_{h}$ and $\langle n\rangle_{h}$, one needs relationships between them and $T_{e h}, T_{i h}, n_{h}$ involved into the right hand sides

$$
n_{h}=\langle n\rangle_{h}-\eta_{c} n_{c}, \quad T_{e, i h}=\frac{0.162 T_{c} / \eta_{c}}{1+\left\langle n T_{i, e}\right\rangle_{h} /\left\langle n T_{e, i}\right\rangle_{h}},
$$

where $\eta_{c}=\frac{0.152\langle n\rangle_{h} T_{c}}{\left\langle n T_{e}\right\rangle_{h}+\left\langle n T_{i}\right\rangle_{h}+0.032 n_{c} T_{c}}$.
Figure 3 shows the time evolution of the parameters $T_{e h}$, $T_{i h}, n_{h}, n_{c}$, and $\Gamma_{c}$, computed for the MGI conditions presented in Sec. IV with the Spitzer-Härm approximation for the electron and ion parallel heat conductions; Figure 4 shows the results obtained with the HFL at $\xi=0.08$ for electrons. One can see that in the former case, the time evolution of $T_{e h}$ is very close to that found above without consideration of the particle convection and density modification induced by the local cooling. With the HFL taken into account, the decay time for the electron temperature is a much longer and in good agreement with the experimental findings, see Refs. 5 and 8 . The saturation of $T_{e h}$ at a level of $100 \mathrm{eV}$, being noticeably higher than that of $50 \mathrm{eV}$ observed in the experiment, is probably due to the crudeness of the used approximation for the term $P$ modeling the radial heat transport. A better agreement is achieved with $\nu$ decreasing in time. Thus, our present results confirm the conclusion of Ref. 9, where a numerical solution of two-dimensional heat balance equations has been found numerically, although with a much

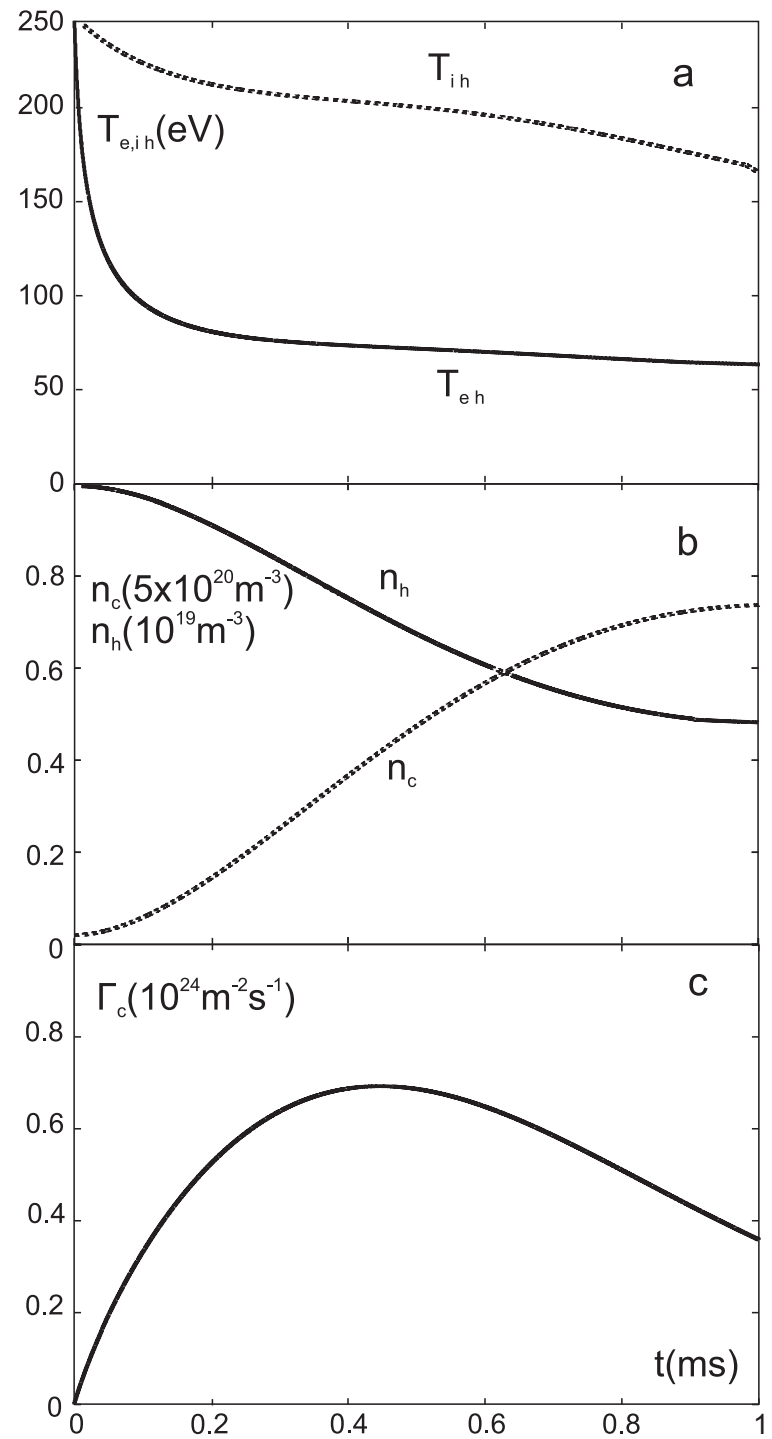

FIG. 3. The time evolution of the electron and ion temperatures (a) and plasma density (b) far away from the cold impurity cloud and at its border, and the density of the parallel particle flux into the cloud (c), computed with $\nu$ $=1000 \mathrm{~s}^{-1}$ and Spitzer-Härm approximation to the parallel heat conductions. 


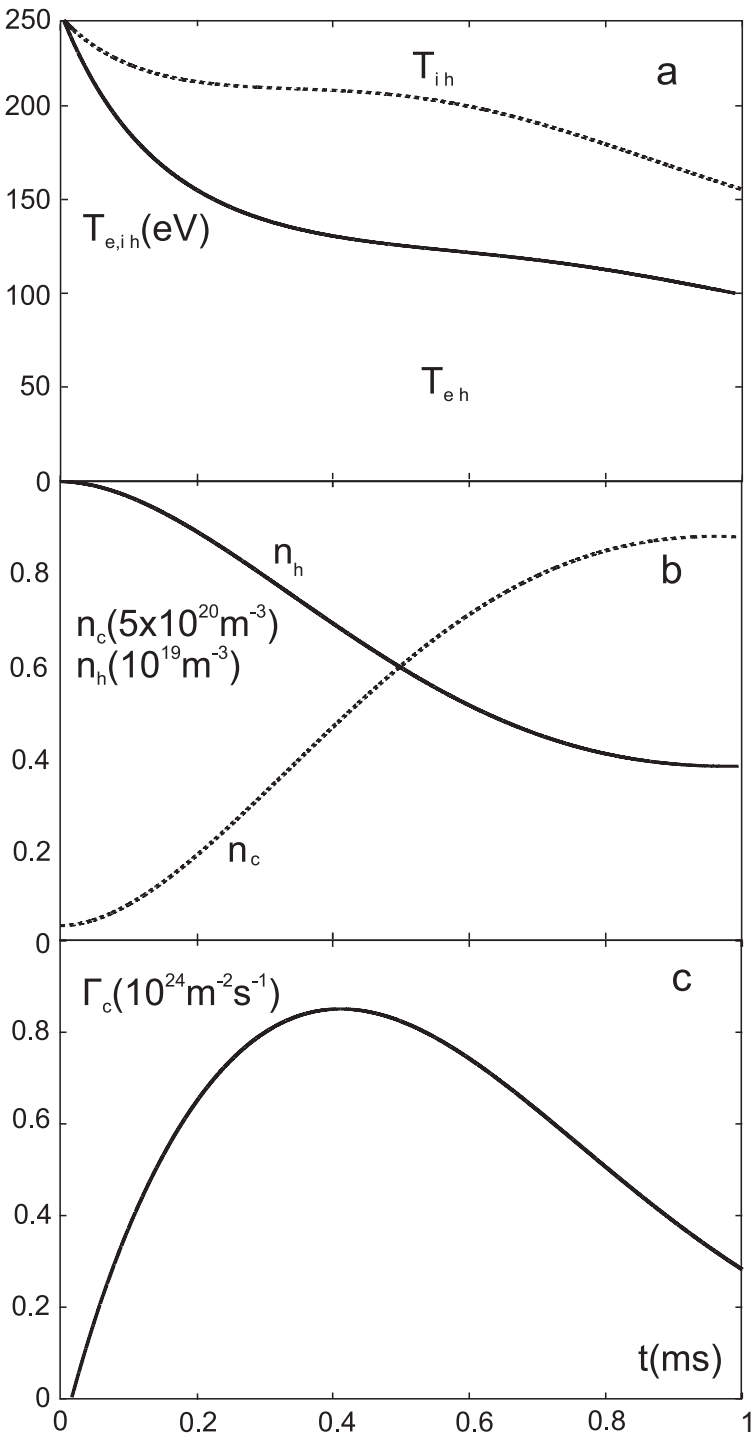

FIG. 4. The time evolution of the electron and ion temperatures (a) and plasma density (b) far away from the cold impurity cloud and at its border, and the density of the parallel particle flux into the cloud (c), computed with $\nu=1000 \mathrm{~s}^{-1}$ and heat flux limit taken into account in the electron heat conduction.

worse convergence in the case with the HFL. Thus, both approaches, the present one with the analytical model for the losses to the cold cloud, and the pure numerical one in Ref. 9 , indicate that the heat flux limit indeed plays an important role for the parameter evolution induced by a strong local cooling.

Another feature, reproducing the experimental findings, is the significant reduction of the plasma density $n_{h}$ far from the cold impurity cloud, see Figures 3(b) and 4(b). A similar behavior has been seen in the edge plasma region by MGI in Alcator C-Mod, see Figure 4 in Ref. 4. This is explained by the pressure equilibration along the magnetic field leading to the condensation of the main plasma particles in the cold impurity cloud.

\section{CONCLUSION}

A simple analytical model for the heat conduction losses along a magnetic surface to the cold impurity cloud near the injection position is elaborated. The model is verified by comparing the results of calculations for the time evolution of the maximum electron temperature on the surface with those obtained by direct numerical solution of non-stationary two-dimensional heat conduction equation, see Ref. 9. The model is extended by including the heat flux limit and a description for the reaction of the plasma density beyond the cold impurity cloud. In agreement with the modeling in Ref. 9 present calculations indicate the importance of HFL for the time evolution of the electron temperature at the plasma edge far from the injection position. The decrease of the edge plasma density found in calculations is caused by the condensation of the main plasma particles in the cold impurity cloud and agrees with observations on Alcator C-Mod tokamak during MGI of argon.

With the present numerically verified analytical approximation for the heat and charged particle losses along magnetic surfaces to the cold impurity cloud, a threedimensional transport equations can be firmly reduced to one-dimensional ones describing the time evolution for the radial profiles of the surface averaged plasma density and temperatures of plasma components. By combining these equations with the "shell" approximation for the impurity spreading from localized sources ${ }^{21}$ and models for the heat balances of the plasma components in the cold cloud, ${ }^{1,2}$ a coherent quasi-three-dimensional approach for modeling of the coherent plasma reaction on a local cooling at the edge is being elaborated now.

Finally, we want to stress the following. Particular calculations in the present paper have been done for the conditions of MGI experiments because they are good documented due to significant interest to this method of disruption mitigation in tokamak devices. Nonetheless, our approach is not intended to modeling of all stages of the plasma shutdown, such as spreading of impurity cloud, thermal and current quenches, but only the initial stage of the edge cooling. This phenomenon is of interest for other applications of impurity injection, in particular, for the questions under which conditions (i) diagnostic impurity beams start to influence the plasma and (ii) plasma cooling can be provoked by impurity seeding done for the purpose of confinement improvement.

${ }^{1}$ M. Z. Tokar and M. Koltunov, Phys. Plasmas 19, 042502 (2012).

${ }^{2}$ M. Koltunov and M. Z. Tokar, Plasma Phys. Controlled Fusion 53, 065015 (2011).

${ }^{3}$ E. M. Hollmann, T. C. Jernigan, M. Groth, D. G. Whyte, D. S. Gray, M. E. Austin, B. D. Bray, D. P. Brennan, N. H. Brooks, T. E. Evans, D. A. Humphreys, C. J. Lasnier, R. A. Moyer, A. G. McLean, P. B. Parks, V. Rozhansky, D. L. Rudakov, E. J. Strait, and W. P. West, Nucl. Fusion 45, 1046 (2005).

${ }^{4}$ R. Granetz, D. G. Whyte, V. A. Izzo, T. Biewer, M. L. Reinke, J. Tery, A. Bader, M. Bakhtiari, T. Jernigan, and G. Wurden, Nucl. Fusion 46, 1001 (2006).

${ }^{5}$ M. Lehnen, A. Alonso, G. Arnoux, N. Baumgarten, S. A. Bozhenkov, S. Brezinsek, M. Brix, T. Eich, S. N. Gerasimov, A. Huber, S. Jachmich, U. Kruezi, P. D. Morgan, V. V. Plyusnin, C. Reux, V. Riccardo, G. Sergienko, M. F. Stamp, and JET EFDA Contributors, Nucl. Fusion 51, 123010 (2011)

${ }^{6}$ B. Unterberg, S. Brezinsek, G. Sergienko, C. C. Chu, P. Dumortier, J. D. Hey, D. Kalupin, A. Kreter, M. Lehnen, A. M. Messiaen, P. Mertens, A. Pospieszczyk, U. Samm, B. Schweer, M. Z. Tokar, G. Van Wassenhove, and TEXTOR-Team, J. Nucl. Mater. 337-339, 515 (2005). 
${ }^{7}$ V. A. Izzo, D. G. Whyte, R. S. Granetz, P. B. Parks, E. M. Hollmann, L. L. Lao, and J. C. Wesley, Phys. Plasmas 15, 056109 (2008).

${ }^{8}$ M. Lehnen, A. Alonso, G. Arnoux, S. A. Bozhenkov, S. Brezinsek, T. Eich, K. H. Finken, A. Huber, S. Jachmich, U. Kruezi, P. D. Morgan, V. V. Plyusnin, C. Reux, V. Riccardo, G. Sergienko, M. F. Stamp, and JET EFDA Contributors, in Proceedings of the 36th EPS Conference on Plasma Physics, Sofia, 2009 ECA, O2.001 (2009), Vol. 33E.

${ }^{9}$ M. Z. Tokar and M. Koltunov, Plasma Phys. Controlled Fusion 55, 045013 (2013).

${ }^{10}$ K. McCormick, S. Fiedler, G. Kocsis, J. Schweinzer, and S. Zoletnik, Fusion Eng. Des. 34-35, 125 (1997).

${ }^{11}$ C. Giroud, R. Barnsley, P. Buratti, I. H. Coffey, M. von Hellermann, C. Jupen, K. D. Lawson, A. Meigs, M. O’Mullane, A. D. Whiteford, K.-D. Zastrow, and JET EFDA Contributors, Nucl. Fusion 47, 313 (2007).

${ }^{12}$ U. Samm, G. Bertschinger, P. Bogen, J. D. Hey, E. Hintz, L. Konen, Y. T. Lie, A. Pospieszczyk, D. Rusbuldt, R. P. Schorn, B. Schweer, M. Tokar, and B. Unterberg, Plasma Phys. Controlled Fusion 35, B167 (1993).

${ }^{13}$ J. Ongena, A. M. Messiaen, B. Unterberg, R. V. Budny, C. E. Bush, K. Hill, G. T. Hoang, G. Jackson, A. Kallenbach, P. Monier-Garbet, D. Mueller, M. Murakami, G. Staebler, F. Ryter, M. Wade, M. Bell, J. Boedo,
G. Bonheure, P. Dumortier, F. Durodie, K. H. Finken, G. Fuchs, B. Giesen, P. Hütteman, R. Jaspers, R. Koch, A. Krämer-Flecken, P. Mertens, R. Moyer, A. Pospieszczyk, A. Ramsey, U. Samm, M. Sauer, B. Schweer, R. Uhlemann, P. E. Vandenplas, G. Van Oost, M. Vervier, G. Van Wassenhove, G. Waidmann, R. R. Weynants, G. H. Wolf, ASDEXUpgrade Team, DIII-D Team, TEXTOR-94 Team, and TFTR Team, Plasma Phys. Controlled Fusion 41, A379 (1999).

${ }^{14}$ L. W. Kantorowitsch and W. I. Krylow, Approximate Methods of Higher Analysis (Interscience Publishers, Inc., New York, 1958), p. 304.

${ }^{15}$ L. Spitzer and R. Härm, Phys. Rev. 89, 977 (1953).

${ }^{16}$ I. Prigogine, Introduction to Thermodynamics of Irreversible Processes, 2nd ed. (Interscience, New York, 1961), p. 53.

${ }^{17}$ S. I. Braginskii, in Reviews of Plasma Physics edited by M. A. Leontovich (Consultants Bureau, New York, 1965), Vol. 1, p. 205.

${ }^{18}$ E. M. Epperlein and R. W. Short, Phys. Fluids B 4, 2211 (1992).

${ }^{19}$ J. F. Luciani, P. Mora, and J. Virmont, Phys. Rev. Lett. 51, 1664 (1983).

${ }^{20}$ R. C. Malone, R. L. McCrory, and R. L. Morse, Phys. Rev. Lett. 34, 721 (1975).

${ }^{21}$ M. Z. Tokar and M. Koltunov, Nucl. Fusion 53, 093014 (2013). 